\title{
Protective Effects of Polysaccharides from Radix Astragali Against Alloxan-induced Oxidative Stress in Diabetic Mice
}

\author{
Ming $\mathrm{Wu}^{\mathrm{a}}$ and Liquan Guo ${ }^{\mathrm{b}}$ \\ Key Laboratory of Grain and Oil Processing of Jilin Province, Jilin Business and Technology College, \\ Changchun, 130062, China \\ awumingl2@sina.com, ${ }^{b} 8413377 @ q q . c o m$
}

\begin{abstract}
Keywords: Oxidative stress; polysaccharides from Radix Astragali; alloxan; diabetic; mice.
Abstract. Oxidative stress has been proposed as an important pathogenic factor in diabetic wound complications. This study investigated the effect of polysaccharides from Radix Astragali (RAP) on alloxan-induced oxidative stress in diabetic mice. Diabetic mice were randomly divided into five groups: diabetic control, positive drug control, low, moderate, and high-dose RAP treated group. Meanwhile, normal mice were used as the control. RAP treated group received different doses of RAP (100, 200, and $400 \mathrm{mg} / \mathrm{kg}$ ) via oral gavage once a day for 4 weeks. The effects of RAP on blood glucose, main antioxidant enzymes and malonaldehyde (MDA) were investigated. The results showed that RAP significantly increased superoxide dismutase (SOD), glutathione peroxidase (GPx) and catalase (CAT) levels in liver and kidney, while decreasing blood glucose levels and MDA levels in liver and kidney in mice. These findings demonstrate that RAP possesses hypoglycemic and protective effect on alloxan-induced oxidative stress in diabetic mice.
\end{abstract}

\section{Introduction}

Diabetes mellitus (DM), one of the major metabolic disorders, is characterized by high blood glucose levels due to the inability of body cells to utilize glucose properly [1]. It is commonly accepted that diabetes is usually accompanied by increased production of reactive oxygen species (ROS) or impaired antioxidant defenses [2]. Hyperglycemia, a prominent clinical feature of diabetes, is a major cause responsible for the increased production of ROS and intensified oxidative stress [3]. Oxidative stress may be a common pathway linking diverse mechanisms for the complications in diabetes [4]. An anti-diabetic drug with antioxidant potential is therefore considered to be very useful and valuable for treatment of diabetes [5]. Due to the huge medical expenditure and complicated pathobiology of diabetes, research has focused on herbal medicine that might improve glucose control and lower the risk of complications [6].

Radix Astragali, the root of Astragalus membranaceus, is one of the oldest and most commonly used of the traditional Chinese medicines [7]. Many pharmacological functions of different components from Radix Astragali have been recognized in "Chinese Pharmacopoeia" [8]. More than 100 compounds exist in Radix Astragali, including flavonoids, polysaccharides, saponins, amino acids and other trace elements [9]. Polysaccharides from Radix Astragali (RAP) is considered to be the principal active ingredients. Recent studies have demonstrated the multiple pharmacological actions of RAP, including anti-tumor, anti-microbial, anti-fatigue, renoprotection and immunomodulatory activities [10,11]. Moreover, studies have also shown that RAP exhibit a strong antioxidant activity [12], which suggesting that it might be beneficial to diabetes-induced oxidative stress. Therefore, the aim of this study was to evaluate the protective effects of RAP on oxidative stress in alloxan-induced diabetic mice.

\section{Materials and Methods}

Chemicals and reagents. Glucose analyzer and strips were purchased from Johnson \& Johnson Co. (USA). Alloxan were purchased from Sigma Chemicals Co. (USA). Glibenclamide were purchased from Sanjing Pharmaceutical Co., Ltd (Harbin, China). The assay kits including superoxide 
dismutase (SOD), glutathione peroxidase (GPx), catalase (CAT) and malonaldehyde (MDA) were purchased from Jiancheng Bioengineering Institute (Nanjing, China).

Laboratory animals. Male Kunming mice (18 to 22 g) were supplied by Experimental Animal Centre of Jilin University (Changchun, China). The animals were kept in a specific pathogen-free animal room maintained at $21 \pm 1{ }^{\circ} \mathrm{C}$ and relative humidity of $55 \pm 10 \%$ with a $12 \mathrm{~h}$ light and dark cycle, and were provided standard rodent diet and tap water.

Plant materials and preparation of polysaccharides. The dried Radix Astragali was obtained from Jilin Province herbal shop and identified by Prof. Zhu Zhu of Jilin Business and Technology College (Changchun, China). The voucher specimens were deposited at the Herbarium of the Northeast Normal University (Changchun, China). The dried samples were ground with a mill and sieved using a 60 -mesh sieve (pore size $0.3 \mathrm{~mm}$ ). The milled powders were sealed in air-tight plastic bags and stored in a desiccator at $-20^{\circ} \mathrm{C}$.

Polysaccharides from Radix Astragali (RAP) were prepared according to the previously published method [13]. In brief, the powders of Radix Astragali extracted with distilled water in a ultrasonic cleaner bath set (KQ2200, Shu-Mei Co., Kunshan, China) at a selected extraction conditions (solid/liquid ratio of 1:20, extraction time of $10 \mathrm{~min}$, ultrasonic power of $65 \mathrm{~W}$ and extraction temperature of $60^{\circ} \mathrm{C}$ ). After filtration to remove debris fragments, the filtrate was concentrated using a speed vacuum concentrator. The proteins in the extract were removed using the Sevag reagent. After removal of the Sevag reagent, the concentrate was mixed with four times its volume of 95\% ethanol before the mixture was maintained overnight at $4{ }^{\circ} \mathrm{C}$ to precipitate polysaccharides.

Induction of diabetes in mice. The freshly prepared solutions of alloxan (200 $\mathrm{mg} / \mathrm{kg}$ body weight) were intraperitoneally injected into the overnight fasted mice. After $72 \mathrm{~h}$, the blood glucose levels were measured by Glucose analyzer. Animals with blood glucose level more than $11.1 \mathrm{mmol} / \mathrm{L}$ were considered as diabetic.

Experiment design. The alloxan-induced diabetic mice were randomly divided into five groups (8 rats per group), and normal mice were used as the control. Normal control (NC) group, normal mice were allowed to free access to a normal diet and treated with distilled water. Diabetic control (DC) group, the diabetic mice were allowed to free access to a normal diet and treated with distilled water. Positive drug control (DPC) group, the diabetic mice were put on a normal diet and treated with glibenclamide solution (4 mg/kg). Low-dose RAP treated (DLRT) group, the diabetic mice were put on a normal diet and treated with RAP solution (100 mg/kg). Moderate-dose RAP treated (DMRT) group, the diabetic mice were put on a normal diet and treated with RAP solution ( $200 \mathrm{mg} / \mathrm{kg})$. High-dose RAP treated (DHRT) group, the diabetic mice were put on a normal diet and treated with RAP solution (400 mg/kg). RAP and glibenclamide were dissolved in $2.0 \mathrm{~mL}$ of distilled water, and the NC and DC groups received the same volume of distilled water. Treatments were administered orally by gavage using a feeding needle once a day for 4 weeks. The doses used in this study were confirmed to be suitable and effective in tested mice according to preliminary experiments.

Biochemical assays. At the end of each week, blood samples were obtained from the tail vein of the overnight fasted mice and their glucose levels were tested by using glucose analyzer. On the last day of experiment, the animals were deprived of food overnight and sacrificed by cervical dislocation. Blood was collected and serum were separated by centrifugation at $3000 \mathrm{rpm}$ for $10 \mathrm{~min}$. Samples were stored at $-70{ }^{\circ} \mathrm{C}$ until assayed. The liver and kidney were removed quickly, cleaned and washed in ice cold normal saline for biochemical study. The levels of SOD, GPx, CAT and MDA in the serum, liver and kidney were measured according to the recommended procedures provided by the commercial assay kits.

Statistical analysis. Data were presented as the mean \pm SD). Statistical comparisons were compared by one-way analysis of variance (ANOVA). The SPSS 12.0 statistical software package (SPSS Inc., Chicago, IL) was used for the analyses. $\mathrm{P}<0.05$ was considered statistically significant. 


\section{Results}

Effects of RAP on the blood glucose of mice. As shown in Fig. 1, blood glucose levels in the NC group remained constant and were significantly decreased when compared with the DPC, DLRT, DMRT, DHRT and DC groups $(\mathrm{P}<0.05)$. After 1 weeks, blood glucose levels in the DPC, DLRT, DMRT and DHRT groups showed a decreasing trend. After 4 weeks, blood glucose levels in the DPC, DLRT, DMRT and DHRT groups were significantly decreased when compared with the DC group $(\mathrm{P}<0.05)$.

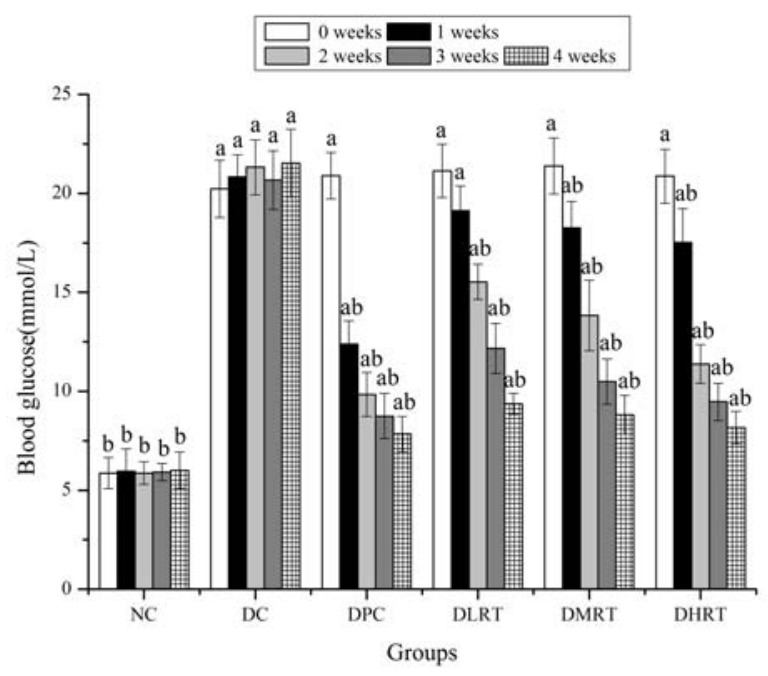

Fig. 1. Effects of RAP on blood glucose of mice. All data are expressed as mean $\pm \mathrm{SD} .{ }^{\mathrm{a}} \mathrm{P}<0.05$ vs normal control (NC) group. ${ }^{b} \mathrm{P}<0.05$ vs diabetic control (DC) group

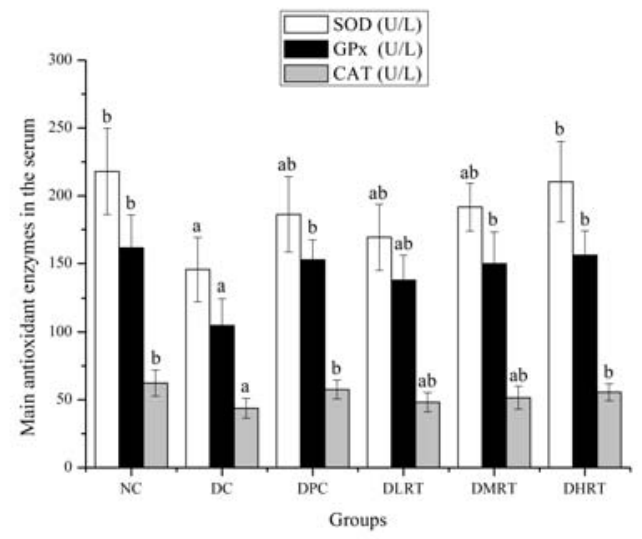

A. serum

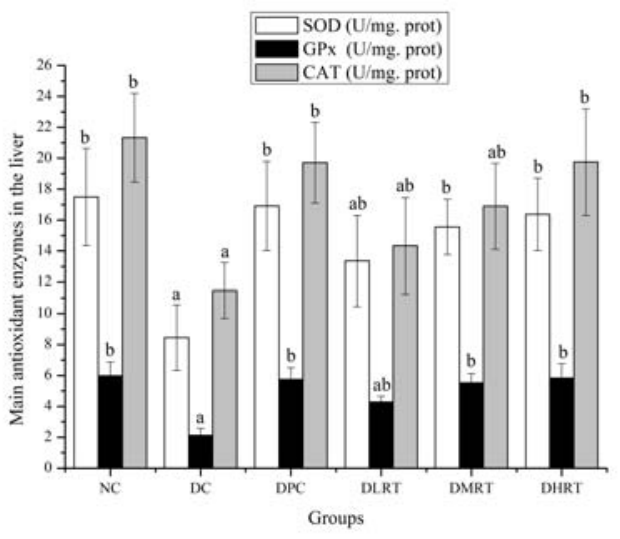

B. liver

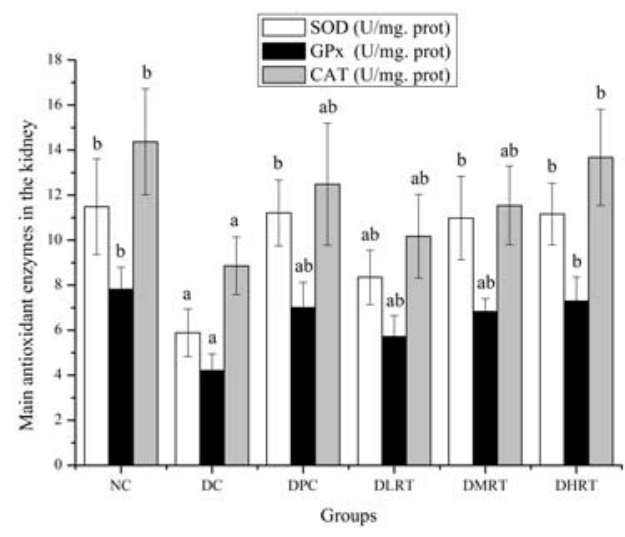

C. kidney

Fig. 2. Effects of RAP on the main antioxidant enzymes in the serum, liver, and kidney of mice. All data are expressed as mean $\pm \mathrm{SD} .{ }^{\mathrm{a}} \mathrm{P}<0.05$ vs normal control (NC) group. ${ }^{\mathrm{b}} \mathrm{P}<0.05$ vs diabetic control

(DC) group 
Effects of RAP on the main antioxidant enzymes in the serum, liver, and kidney of mice. As shown in Fig. 2A, the SOD levels in the serum in the DC, DPC, DLRT and DMRT groups, the GPx levels in the serum in the DC and DLRT groups, as well as the CAT levels in the serum in the DC, DLRT and DMRT groups were significantly decreased when compared with the NC group $(\mathrm{P}<0.05)$. However, the SOD, GPx and CAT levels in the serum in the DPC, DLRT, DMRT and DHRT groups were significantly increased when compared with the DC group $(\mathrm{P}<0.05)$. As shown in Fig. $2 \mathrm{~B}$, the SOD and GPx levels in the liver in the DC and DLRT groups, as well as the CAT levels in the serum in the DC, DLRT and DMRT groups were significantly decreased when compared with the NC group $(\mathrm{P}<0.05)$. However, the SOD, GPx and CAT levels in the liver in the DPC, DLRT, DMRT and DHRT groups were significantly increased when compared with the $\mathrm{DC}$ group $(\mathrm{P}<0.05)$. As shown in Fig.2C, the SOD levels in the kidney in the DC and DLRT groups, as well as the GPx and CAT levels in the kidney in the DC, DPC, DLRT and DMRT groups were significantly decreased when compared with the NC group $(\mathrm{P}<0.05)$. However, the SOD, GPx and CAT levels in the kidney in the DPC, DLRT, DMRT and DHRT groups were significantly increased when compared with the DC group $(\mathrm{P}<0.05)$. Effects of RAP on the MDA in the serum, liver and kidney of mice. As shown in Fig. 3, the MDA levels in the serum in the DC, DPC, DLRT, DMRT and DHRT groups, MDA levels in the liver in the DC and DLRT groups, as well as the MDA levels in the kidney in the DC, DPC, DLRT and DMRT groups were significantly increased when compared with the NC group $(\mathrm{P}<0.05)$. However, the MDA levels in the serum, liver and kidney in the DPC, DLRT, DMRT and DHRT groups were significantly decreased when compared with the DC group $(\mathrm{P}<0.05)$

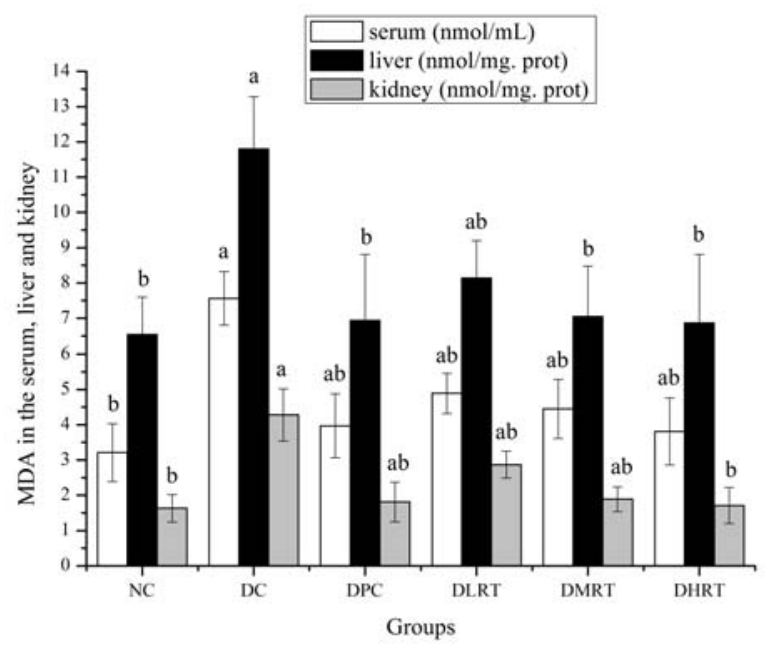

Fig. 3. Effects of RAP on the MDA in the serum, liver and kidney of mice. All data are expressed as mean $\pm \mathrm{SD} .{ }^{\mathrm{a}} \mathrm{P}<0.05$ vs normal control (NC) group. ${ }^{\mathrm{b}} \mathrm{P}<0.05$ vs diabetic control (DC) group

\section{Discussion}

Alloxan monohydrate is one of the chemical agents used to induce diabetes mellitus in animals since it produces oxygen radicals in the body, which causes pancreatic injury [14]. Intraperitoneal administration of alloxan ( $200 \mathrm{mg} / \mathrm{kg}$ body weight) effectively induced diabetes mellitus in normal mice as reflected by glycosuria, hyperglycaemia, polyphagia, polydipsia and body weight loss compared with normal mice [15]. In this study, alloxan-induced diabetic mice presented obvious hyperglycemic symptoms, and RAP treatment for 4 weeks showed significant reduction in blood glucose levels. These results indicated that RAP possesses hypoglycemic effects.

Oxidative stress has been proposed as an important pathogenic factor in diabetic wound complications. While on the one hand hyperglycemia engenders free radicals, on the other hand it also impairs the endogenous antioxidant defense system in many ways during diabetes [4]. Oxidative stress can damage several biomolecules like proteins, lipids and DNA, thereby leading to the inactivation of enzymes affecting DNA integrity and cellular membrane composition [16]. Thus, the 
ideal anti-diabetic drug should combine both hypoglycemic and antioxidant properties. Liver and kidney are the most important organs, which play a pivotal role in regulating various physiological processes in the body [17]. In this study, the results showed that RAP could significantly increase SOD, GPx and CAT levels, decrease MDA levels in liver and kidney in mice, which suggest that RAP are able to upregulate main antioxidant enzyme activities and reduce lipid peroxidation to protect against alloxan-induced oxidative stress in diabetic mice. These effects might be due to its antioxidant property of RAP.

\section{Summary}

The findings of this study demonstrate that RAP possesses hypoglycemic and protective effect on alloxan-induced oxidative stress in diabetic mice. The antioxidant property of RAP may also play a protective role in the development of diabetes. RAP may be of use as an anti-diabetic drug. However, further study is needed to elucidate hypoglycemic mechanism of RAP at the cellular and molecular levels.

\section{References}

[1] W.L. Xue, X.S. Li, J. Zhang, Y.H. Liu, Z.L. Wang and R.J. Zhang: Asia Pac. J. Clin. Nutr Vol. 16 (2007), p. 422.

[2] A.C. Maritim, R.A. Sander and J.B. Watkins: J. Biochem. Mol. Toxicol Vol. 17 (2003), p. 24

[3] F. Song, X. Qi, W. Chen, W. Jia, P. Yao, A.K. Nussler, X. Sun and L. Liu: Eur. J. Nutr Vol. 46(2007), p. 61

[4] S.X. Xue, X.M. Chen 1, J.X. Lu and L.Q. Jin: Carbohyd. Polym Vol. 75(2009), p. 415

[5] H. Karki, K. Upadhayay, H. Pal and R. Singh: Int. J. Green Pharm Vol. 8(2014), p. 77

[6] W. Zhu, M. Chen, Q. Shou, Y. Li and F. Hu: Evid. Based Complement. Alternat. Med Vol. (2011), p. 468529

[7] X. Du, X. Chen, B. Zhao, Y. Lv, H. Zhang, H. Liu, Z. Chen, Y. Chen and X. Zeng: FEMS Immunol. Med. Microbiol Vol. 63(2011), p. 228

[8] Z.Y. Zhu, R.Q. Liu, C.L. Si, F. Zhou, Y.X. Wang, L.N. Dingo, C. Jing, A.J. Liu and Y.M. Zhang: Carbohyd. Polym Vol. 85(2011), p. 895

[9] S.C. Kang, H.J. Kim and M.H. Kim: Biol. Trace. Elem. Res Vol. 68(2013), p. 151

[10]B. Yang, B. Xiao and T. Sun: Int. J. Biol. Macromol Vol. 62(2013), p. 287

[11]H.J. Kwon, J. Hwang, S.K. Lee and Y.D. Park: J. Nat. Med Vol. 67(2013), p. 850

[12]R. Jia, L. Cao, P. Xu, G. Jeney and G. Yin: Fish Physiol. Biochem Vol. 38(2012), p. 871

[13] M. Wu, C.F. Ma, J.M. Kan and L.Q. Guo: Adv. Mater. Res Vol. 549 (2014), p. 864

[14] Y. Liu, Z.J. Chen, Y.T. Liang, L.Y. Cui and D. Zheng: Chem. Res. Chinese U Vol. 25 (2009), p. 681

[15]K. Rajagopal and K. Sasikala: Singapore Med. J Vol. 49 (2008), p. 137

[16] M.M. Alam, D. Meerza and I. Naseem: Life Sci Vol. 109 (2014), p. 8

[17]X.M. Li: Int. J. Biol. Macromol Vol. 40 (2007), p. 461 